\title{
Simple and Stable Algorithm for Parameter Identification in Heat Conduction Problems
}

\author{
Junli Zhang ${ }^{1, a}$, Suhua Wang ${ }^{2, b}$, Guichun $\operatorname{Han}^{3, c}$ \\ ${ }^{1,3}$ School of Mathematics, Inner Mongolia University for the Nationalities, Tongliao, 028043, China \\ ${ }^{2}$ Qinhuangdao Institute of Technology,Qinhuangdao,066100,China \\ a jl_zhang7706@163.com, bsuhua_1013@sina.com ${ }^{\mathrm{c}}$ hanguicun@163.com
}

Keywords: heat conduction equation; inverse problem; parameter identification; constraint condition

\begin{abstract}
Parameter identification in heat conduction problems is an ill-posed problem in the sense of Hadamard, this means that small errors in the measurement may cause very large errors in the numerical result. In this paper, a regularization method for inverse problem of determination of unknown parameter in heat Conduction problems is presented. The approach of the proposed method is to add constraint condition of derivative and get a well-posed operator equation which can approximate the ill-posed problem discussed. The numerical results show that our algorithm is stable and precise.
\end{abstract}

\section{Introduction}

A great amount of research work has focused on the development of appropriate strategies for solving inverse problems in many fields of application ranging [1-8]. In this paper, we apply a simple and stable algorithm for solving inverse problems in heat conduction equations and demonstrate merit of the algorithm through numerical tests.

Wang Ping and Kewang Zheng considered the question of determining an unknown source in the heat equation from over-specified data [2]. Zhilin Li \& Kewang Zheng considered the question of determining an unknown parameter in the heat equation [3]. In a general way, we transform inverse problems in heat conduction equations into solving the following Volterra operator equation of the first type.

$$
(A z)(t):=\int_{0}^{t} z(t) d \tau=f(t), t \in[0, T]
$$

However, (1) is an ill-posed problem in the sense of Hadamard, this means that small errors in the measurement may cause very large errors in the numerical result [4].Thus some kind of regularization method must be utilized to obtain the stable numerical resolution of the problem (1).

\section{Regularization algorithm}

Up to the present, lots of research work has been done for solving ill-posed problems. The problem (1) can be solved by Tikhonov regularization algorithms and regularization strategies supplied in [4] theoretically. In this paper, we will solve it employing a Simple and Stable Algorithm.

Pay attention to (1), the precise $f(t) \in H^{1}$ is approximately known with a noise level $\delta$ : $\left\|f_{\delta}-f\right\| \leq \delta$. In fact, we will solve the following equation

Discretize (2) primarily.

$$
A z(t)=f_{\delta}(t)
$$

Let $h=\frac{T}{n}$ be step length on $t$ axis and $\tau$ axis and define a grid $W_{t} \times W_{\tau}$ in $[0, T]$.

$$
W_{t}=\left\{t_{i} \mid t_{i}=i h, i=0,1,2, \ldots, n\right\} ; W_{\tau}=\left\{\tau_{i} \mid \tau_{i}=j h, j=0,1,2, \ldots, n\right\} .
$$


so $\int_{0}^{t_{i}} z(\tau) d \tau=f_{\delta}\left(t_{i}\right), \quad i=1,2, \ldots, n$. then,

$$
\int_{t_{i-1}}^{t_{i+1}} z(\tau) d \tau=f_{\delta}\left(t_{i+1}\right)-f_{\delta}\left(t_{i-1}\right), i=1,2, \ldots, n-1
$$

Apply Simpson formula, that is

$$
\frac{h}{3}\left[z\left(t_{i+1}\right)+4 z\left(t_{i}\right)+z\left(t_{i-1}\right)\right]=f_{\delta}\left(t_{i+1}\right)-f_{\delta}\left(t_{i-1}\right), \quad i=1,2, \ldots, n-1 .
$$

Express it by matrix, then

$$
\left(\begin{array}{cccccc}
1 & 4 & 1 & 0 & \ldots & 0 \\
0 & 1 & 4 & 1 & \ldots & 0 \\
\vdots & \vdots & \ddots & \ddots & \ldots & 0 \\
0 & 0 & 0 & 1 & 4 & 1
\end{array}\right)\left(\begin{array}{c}
\mathrm{z}\left(t_{0}\right) \\
\mathrm{z}\left(t_{1}\right) \\
\vdots \\
\mathrm{z}\left(t_{n}\right)
\end{array}\right)=\frac{3}{h}\left(\begin{array}{c}
f_{\delta}\left(t_{2}\right)-f_{\delta}\left(t_{0}\right) \\
f_{\delta}\left(t_{3}\right)-f_{\delta}\left(t_{1}\right) \\
\vdots \\
f_{\delta}\left(t_{n}\right)-f_{\delta}\left(t_{n-2}\right)
\end{array}\right)
$$

Take the matrix at the ring-hang as $A_{1}$, which approximates the operator in (2), and the vectors above written $z_{h}(t)$ and $f_{\delta}^{h}$ respectively. As a result,

$$
A_{1} z_{h}(t)=f_{1}^{h}, 0<t<T .
$$

This is an underdetermined system of equations, and it is caused by the ill-posed problem (2). If $z\left(t_{0}\right)$ and $z\left(t_{n}\right)$ are known, then

$$
\left(\begin{array}{ccccc}
4 & 1 & 0 & \cdots & 0 \\
1 & 4 & 1 & \cdots & 0 \\
\vdots & \ddots & \ddots & \ddots & \vdots \\
0 & 0 & 0 & 1 & 4
\end{array}\right)\left(\begin{array}{c}
\mathrm{z}\left(t_{1}\right) \\
\mathrm{z}\left(t_{2}\right) \\
\vdots \\
\mathrm{z}\left(t_{n-1}\right)
\end{array}\right)=\frac{3}{h}\left(\begin{array}{c}
f_{\delta}\left(t_{2}\right)-f_{\delta}\left(t_{0}\right)-z\left(t_{0}\right) \\
f_{\delta}\left(t_{3}\right)-f_{\delta}\left(t_{1}\right) \\
\vdots \\
f_{\delta}\left(t_{n}\right)-f_{\delta}\left(t_{n-2}\right)-z\left(t_{n}\right)
\end{array}\right)
$$

The equation above is well-posed and exists unique solution, but $z\left(t_{0}\right)$ and $z\left(t_{n}\right)$ are unknown, so we can't solve (5).

We add constrained information of derivative

$$
\int_{0}^{t_{k}} z(\tau) d \tau=f_{\delta}^{h}\left(t_{k}\right), k=n-1, n
$$

Applying duplicate trapezoid formula to approximate (6), then

$$
\left(\begin{array}{cccccc}
1 & 2 & 2 & \cdots & 1 & 0 \\
1 & 2 & 2 & \cdots & 2 & 1
\end{array}\right)\left(\begin{array}{c}
z\left(t_{0}\right) \\
z\left(t_{1}\right) \\
\vdots \\
z\left(t_{n}\right)
\end{array}\right)=\frac{2}{h}\left(\begin{array}{c}
f_{\delta}^{h}\left(t_{n-1}\right) \\
f_{\delta}^{h}\left(t_{n}\right)
\end{array}\right)
$$

Let

$$
A_{2} z_{h}(t)=\left(f_{2}^{h}\right)
$$

In order to improve boundary precision, when $i=1$ in (5), we change it into the following equation

$$
\int_{t_{0}}^{t_{2}} z(\tau) d \tau=f_{\delta}\left(t_{2}\right)-\frac{1}{n^{2}} f_{\delta}\left(t_{1}\right)
$$

then $A_{1}$ is displaced by $A_{3}$ and $f_{1}^{h}$ is displaced by $f_{3}^{h}$.

Put (3),(7) and (8) together, then

$$
A_{h} z_{\delta}^{h}=f_{\delta}^{h}, A_{h}=\left(\begin{array}{c}
A_{3} \\
A_{2}
\end{array}\right), f_{\delta}^{h}=\left(\begin{array}{c}
f_{1}^{h} \\
f_{3}^{h}
\end{array}\right) .
$$


The $A_{h}$ is a nonsingular matrix and its condition number is not large, so (9) is well-posed and exists unique solution. We take (9) as a regularization algorithm for (2), we define (9) for $A C$ regularization method. Numerical experiments to test the effectiveness of this algorithm which will be discussed in the following section.

\section{Numerical Results}

About heat conduction equations in paper [2],

$$
\begin{gathered}
u_{t}=u_{x x}+a(t) u, \quad x>0, \quad 0<t<T . \\
u(x, 0)=0, \quad x>0 . \\
u(0, t)=\varphi(t), \quad f(0)=0,0<t<T . \\
-u_{x}(0, t)=g(t), \quad g(t)>0, \quad 0<t<T .
\end{gathered}
$$

where $\varphi(t)$ and $g(t)$ are assumed to be known and strictly increasing functions, we will find $a(t)$, this is an inverse problem. We will apply $A C$ methods to determine coefficient $a(t)$.

Fig. 1 and Fig.2 apply Tikhonov regularization algorithms and our regularization algorithms respectively to get approximate solution and to fit the exact solution when $n$ equals 100 . The numerical results show our algorithms are much more accurate compared with Tikhonov regularization algorithms at two endpoints.

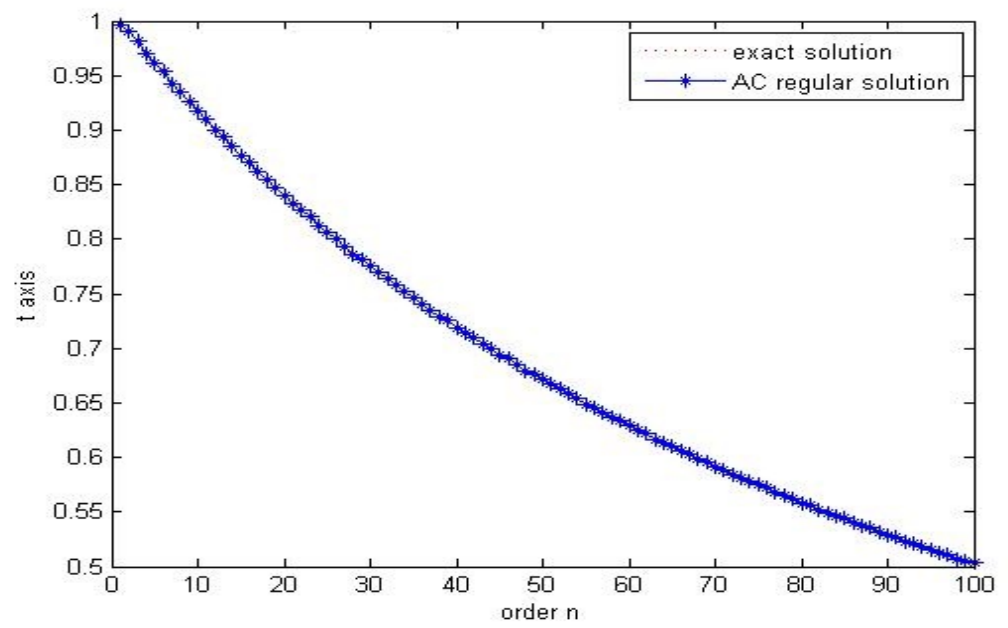

Fig.1: The exact and regular solution (AC), $\left(n=100, \delta=10^{-5}\right)$

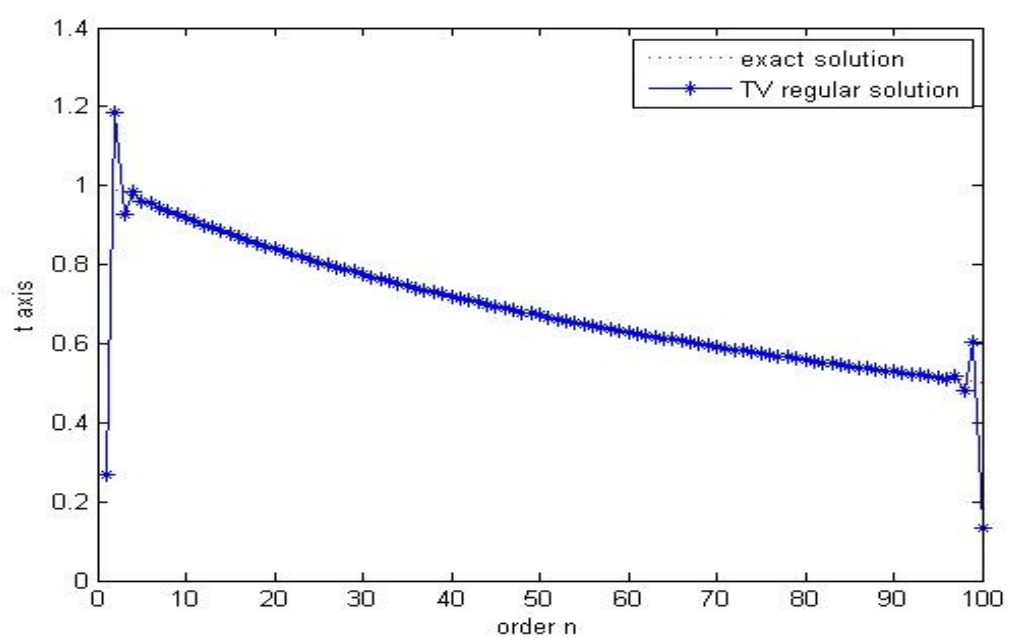

Fig.2: The exact and regular solution (TV), $\left(n=100, \delta=10^{-5}\right)$

Table. applies our regularization algorithms and Tikhonov regularization algorithms based on Morozov discrepancy principle to determinate source respectively with the different scale problems. 
Numerical experiment results show our regularization algorithms $(A C)$ is more accurate than Tikhonov regularization algorithms.

Table. Numerical stability of our algorithms and Tikhonov algorithms $\left(\delta=10^{-5}\right)$

\begin{tabular}{c|c|c|c|c|c|c|c}
\hline$n$ & 100 & 200 & 500 & 1000 & 1500 & 2000 & 3000 \\
\hline$\frac{\left\|f_{T V}^{h}-f_{T}^{h}\right\|_{2}}{\left\|f_{T}^{h}\right\|_{2}}$ & 0.1695 & 0.0849 & 0.0538 & 0.0386 & 0.0328 & 0.0304 & 0.0306 \\
\hline$\frac{\left\|f_{A C}^{h}-f_{T}^{h}\right\|_{2}}{\left\|f_{T}^{h}\right\|_{2}}$ & $9.405 \mathrm{e}-4$ & 0.0014 & 0.0035 & 0.0070 & 0.0106 & 0.0144 & 0.0214 \\
\hline
\end{tabular}

In conclusion, the numerical results in TABLE above show that $A C$ algorithms is more accurate and stable compared with Tikhonov regularization algorithms, and from Fig1 and Fig2, we can see regularization algorithms based dynamical systems methods is effective. So we can regard dynamical systems methods as a regularization algorithm for ill-posed problems in scientific computing when the scale of problem is not too larger, it provides an effective tool for practitioners.

\section{Acknowledgment}

This work was financially supported by the science fund of Inner Mongolia University for the Nationalities (grant No. NMD1305) and the National Natural Science Foundation of China (No. NJZY13159).

\section{References}

[1] M.X. Wang ,Tingyan Xiao: Traffic Engineering, 5(2009), p. 222-225.

[2] Z.L. Li, K.W. Zheng: Electronic Journal of Differential Equations,1(1997), p.203-209.

[3] P.W, K.W. Zheng: Electronic Journal of Differential Equations,3(1999), p.119-125..

[4] T.Y. Xiao, Y.F. Wang ,S.G. Yu: Beijing. Science Press, 2003.

[5] A.G. Fatullayev: Applied Mathematics and Computation, 152(2004), p. 659-666.

[6] T.Y. Xiao, J.L. Zhang: Chinese Journal of Computational Physics, 3(2008), p. 335-343.

[7] J.L. Zhang, H.Y. Yu: 2011 Matrices and Operators, Vol.1, p.193-196.

[8] Y.F. Wang, T.Y. Xiao: Inverse Problems, 17(2001), p.281-291. 